\title{
ESTUDIOS COMPARATIVOS DE LOS ETOGRAMAS DE OTARIA FLAVESCENS, ARCTOCEPHALUS AUSTRALIS Y OTROS OTARIIIDOS (MAMMALIA)
}

\author{
RAUL VAZ-FERREIRA \\ SILVANA VALLEJO \\ MARIO D. HUERTAS
}

\section{INTRODUCCION}

Se realizaron estudios sobre el comportamiento de Arctocephalus australis y Otaria flavescens en 92 viajes a las islas y loberias del Uruguay, efectuándose unas 720 horas de observación sobre A. australis y 600 horas sobre $O$. flavescens. También uno de los autores (R. V. F.) efectuó observaciones sobre Arctocephalus pusillus en Seal Island, del Cabo Buena Esperanza, y en los criaderos terrestres de esta especie en Cape Cross (Sudáfrica) y también estudios sobre Callorhinus ursinus y Eumetopias jubatus en las Islas Pribilof (Alaska).

Se consideran, por otra parte, estudios etológicos realizados por otros autores, que alcanzan la casi totalidad de las 14 especies reconocidas de otaríidos. En este trabajo, nos restringimos a comportamientos territorial, agonístico, precopulatorio, copulatorio e de bienestar y/o termorregulatorio.

\section{COMPORTAMIENTO TERRITORIAL Y EXPRESIONES AGONISTICAS RELACIONADAS}

Es característico de los otaríidos el establecimiento de los machos, durante la época reproductora, en áreas precisas, que defienden de otros machos y que constituyen los llamados territorios (o áreas de influencia, Miller, 1975).

Las áreas o zonas elegidas para el establecimiento o fundación de territorio, así como su tamaño y delimitación varia según la especie.

Otaria flavescens suele ubicar sus territorios en orillas llanas, con arena y piedras, o a veces, en áreas de piedras planas.

El área ocupada por cada macho de esta especie suele variar entre 25 y $60 \mathrm{~m} 2$. Datos al respecto han sido aportados por Gentry (1975), para Eumetopias jubatus (205 m2) y para Arctocephalus forsteri $\left(23 \mathrm{~m}^{2}\right)$.

En el Uruguay accidentes topográficos pueden contribuir a delimitar los territorios, no siendo estos habitualmente sobrepasados si tienen una altura mayor de un metro.

Departamento de Zoología Vertebrados, Faculdad de Humanidades y Ciencias, Tristán Narvaja 1674, Montevideo, Uruguay. 
En zonas en las cuales no existen señas topográficas se mantiene una distancia interindividual más o menos constante.

A. australis elije preferentemente áreas rocosas cercanas a la orilla, o con sombra o charcos permanentes. Esto posibilita la utilización de un área de territorios más alejada o con cierta independencia del mar, pero que permiten la disponibilidad de lugares aptos para la supervivencia de los cachorros y la resistencia de los machos territoriales en tierra.

El establecimiento de territorios comienza siempre antes del arribo de las hembras. En $A$. australis, comienza a partir de la primera semana de noviembre hasta aproximadamente el 10 diciembre, y en $O$. flavescens desde el 15 de diciembre hasta el 6 de enero.

La adherencia a los territorios por regla es máxima durante el climax de la estación reproductora, y en general, los machos territoriales no abandonam estas áreas ni aún durante las horas de más calor o de crecida de la marea. Para $A$. australis hemos constatado una permanencia de más de 50 días en el territorio. Gentry (op. cit.) ha estabelecido una media de 46 días de permanencia en território para $E$. jubatus y de 38 para $A$. forsteri.

Más adelante en la estación, los machos tanto de Otaria como de A. australis hacen incursiones al agua, abandonando temporaria o definitivamente el área territorial. En este último caso suelen ser substituídos por machos más jóvenes.

Los límites de los territorios son en general fluctuantes durante el correr de la estación, y sufren ajustes a lo largo de la misma. Estos están sometidos a la constante presión de los machos que arriban al área, o de aquellos que intentan conquistar o expandir territorio.

Además de la lucha, para los otariidos, el territorio se define o delimita por medio de despliegues simbólicos, que incorporan en forma ritualizada elementos que forman parte de la lucha física.

Estos despliegues se integran con elementos motores, acústicos y visuales (principalmente posturas), de diferente intensidad e importancia en los distintos integrantes de la familia. Durante estos despliegues raramente ocurren mordidas, y cuando estas se dan, son rápidas, y para el caso de $O$. flavescens se localizan especialmente en la cara.

Es común y periódica la vocalización de redefinición territorial, que en Otaria consiste en desafíos compuestos por rugidos de medio segundo de duración, con frecuencias de hasta $6 \mathrm{KHz}$ (Vaz-Ferreira, 1981). En A. australis, además de los elementos vocales, el ritual de redefinición se integra con elementos locomotores, consistentes en aproximación de los vecinos, luego de lo cual se disponen verticalmente los hocicos, permaneciendo así unos instantes y luego retrocediendo ambos (Vaz-Ferreira \& Vallejo, 1981).

En general, los otariidos expanden, conquistan y defienden territorio mediante lucha física. En Otaria la lucha física se formaliza con mordeduras en cabeza y cuello, y con menos frecuencia en la base de los miembros anteriores y el resto del cuerpo. La lucha puede durar varios minutos, y se resuelve con la huída de uno de los contrincantes, en la 
mayoría de los casos el macho invasor. En $A$. australis, la lucha tiene períodos activos, con mordidas y sacudidas, y períodos pasivos durante los cuales los contrincantes se inmovilizan mutuamiente conteniendo pecho contra pecho, o sujetándose entre sí con la boca. Cuando uno de ellos intenta abandonar la posición de quietud, se formaliza nuevamente un período activo (Vaz-Ferreira \& Vallejo, op. cit.). La conclusión de la lucha física está dada por la huída de uno de los contricantes en la mayoria de los casos. Las heridas más típicas ocurren especialmente en la cara y el resto de la cabeza, zonas posteriores del cuerpo, y dorso y base de los miembros.

Otra actividad realizada por los machos territoriales es la relacionada con la retención de hembras. Esta actividad es más o menos desarrollada en las distintas especies. En los casos más extremos implica una atracción activa de las hembras al territorio, y un patrullaje contínuo a fin de evitar la evasión de hembras, como se da en Callorhinus ursinus de acuerdo con Bartholomew \& Hoel, 1953. En la mayoría de los casos la actividad de retención de hembras se restringe a evitar que éstas abandonen el territorio o a retener a las transeúntes. La forma más común de retención se da por interposición del cuerpo del macho entre la hembra y el límite territorial. En Otaria se registran maniobras de rodeo por parte de los machos y sobre todo de retención individual, que se expresa mediante obstrucción al avance de la hembra utilizando el cuello y la cabeza, y ondulando lentamente el tren anterior. Las maniobras de retención son más violentas si el macho de Otaria implicado tiene solamente una hembra. En este último caso el macho puede perseguir a la fugitiva fuera de los límites territoriales, y atraerla violentamente con los dientes. A. australis impide por diferentes procedimientos el alejamento de las hembras: por interposición del cuerpo, por contactos de hocicos más o menos violentos, por erección de vibrisas, y más excepcionalmente mediante mordeduras o persecusión fuera del territorio (Vaz-Ferreira \& Vallejo, op. cit.).

\section{CARACTERES DE LA LUCHA Y OTRAS EXPRESIONES AGONISTICAS}

En Otaria flavescens la mayor parte de las luchas efectivas son de corta duración, 5 segundos o menos, y consisten en agresión frontal o lateral, en la que ambos individuos se muerden, con sacudidas muy rápidas de la cabeza, la cara y la parte superior del cuello. Las mordeduras en otras partes del cuerpo son menos frecuentes. Las luchas entre hembras consisten sobre todo en amenazas de boca abierta y desafíos vocales.

En Otaria, la sumisión se expresa por huída, no se han descrito vocalizaciones o expresiones faciales o somáticas de sumisión.

En Otaria flavescens existen fenómenos agonisticos peculiares a los machos subadultos, consistentes en el ataque en grupo a las estructuras reprodutoras, o el rapto y eventual agresión o matanza de los cachorros 
(infanticidio) (Vaz-Ferreira, 1965), fenómeno registrado también ulteriormente en Neophoca cinerea por Marlow, 1975.

El comportamiento agonístico de Arctocephalus australis se expresa por lucha precedida de enfrentamiento con cuello y cabeza levantados y mordeduras en la cabeza, cuerpo y miembros anteriores, que alternan con posiciones de descanso en que cada uno retiene al adversario por áreas simétricas del cuerpo; pasada una etapa de retención, las mordeduras activas se reiteran, y la secuencia llega a durar desde algunos minutos a más de media hora.

En Arctocephalus australis existen expresiones posturales y vocales indicativas de sumisión, éstas se manifestan, ya sea sin lucha, cuando un individuo de ubicación socio-reproductora baja enfrenta o otro superior, cuando una hembra con o sin cachorro quiere pasar entre territorios, o después de librar una lucha en que un individuo resulta vencido. Desde el punto de vista postural, la sumisión se expresa bajando la cabeza y abriendo la boca a veces en forma reiterada, mientras la cabeza se orienta hacia los posibles agresores, al mismo tiempo que se efectúan giros bruscos del cuerpo. Simultáneamente ocurren expresiones vocales consistentes en alaridos agudos. Este comportamiento coincide con el de otros Otariidae estudiados por otros autores.

\section{COMPORTAMIENTO PRECOPULATORIO Y COPULATORIO}

La existencia de un comportamiento precopulatorio ha sido descrita para varias especies de otariidos: A. gazella, - Paulian, 1964 -; Callorhinus ursinus, - Peterson, 1965 (citado por Miller, 1975) -; Zalophus californianus, - Peterson \& Bartholomew, 1967 -; A. forsteri, - Miller, 1974 -; Neophoca cinerea y Phocarctos hookeri, - Marlow, op. cit. -; Eumetopias jubatus, - Sandegreen, 1975 - A. austratis, - Vaz-Ferreira \& Vallejo, 1981 —. Este puede ser simplemente desarrollado por el macho mediante exploración vulvar, o estar constituído por un complejo conjunto de señales o despliegues realizados por la hembra en estro y que pueden ser seguidos por una respuesta más o menos compleja del macho. Un despliegue precopulatorio complejo ha sido descrito por Sandegreen (op. cit.), para Eumetopias jubatus, este despliegue se caracteriza por una serie de movimientos y posturas, que pueden lograr la estimulación del macho.

En Otaria, a menudo se registra olfación y/o contacto de hocicos, olfación vulgar, contacto de cabezas y frotamiento de cuellos. En esta especie la hembra puede realizar moderadas ostentaciones de acercamiento de cuerpos, pero no existe ostentación o invitación periestral como la que se apresenta en Zalophus californianus, de acuerdo con Peterson \& Bartholomew (op. cit.), y en Callorhinus ursinus, según Peterson (op. cit.).

En $A$. australis, es común la búsqueda de hembras en estro por parte de los machos, mediante olfación de la cara y de la región vulvar. Es poco frecuente y poco exitosa en esta especie la búsqueda de machos 
por parte de hembras en estro, y cuando ésta ocurre la hembra la expresa siguiendo al macho o girando alrededor de éste.

La hembra de Otaria evagina la vulva poco antes del coito, y el macho progresivamente va adoptando una posición de cabalgamiento, que en ciertos casos es resistida por la hembra, que levanta rígidamente la cola y entrecruza uno de los miembros posteriores. Luego de determinado tiempo la cópula es aceptada por la hembra, mediante la separación de los miembros posteriores. Luego de la intromisión y de balanceo hacia los lados comienzan las sacudidas pélvicas. Es frecuente que la hembra muerda el cuello del macho, lo que se acompaña de un incremento en frecuencia e intensidad de las sacudidas pélvicas. Durante la cópula el macho sube y baja alternativamente la cabeza. La cópula de $O$. flavescens ocurre normalmente en el territorio donde la hembra está con su cachorro, aunque a veces puede darse fuera del territorio establecido. Durante ésta, la hembra de Otaria se halla apoyada sobre el vientre, el macho puede estar erguido o acostado sobre la hembra, presionándola con el cuello. Esta posición durante la cópula se observa en líneas generales también en $A$. austratis, - Vaz-Ferreira y Vallejo, op. cit. -, A gazella, - Paulian, op. cit. - . En A. australis y A. forsteri, los intentos de intromisión suelen ser varios, debido principalmente a las tentativas de rechazo por parte de la hembra. En A. australis, éstas se componen principalmente de huída, o rechazo mediante vocalizaciones, amenaza de boca abierta y a veces, mordeduras. Una vez lograda la intromisión el macho comienza las sacudidas pélvicas. Hacia el final de la cópula la hembra incrementa los esfuerzos por escapar y la actitud agresiva hacia el macho.

Las responsables aparentes de la finalización de la cópula son, en la mayoría de los casos, las hembras. Esto lo hemos observado en O. flavescens y A. australis, especies en las cuales la desmonta coincide con un notorio incremento de las mordeduras y de la actitud agresiva de la hembra en general. La agresión desarrollada por la hembra hacia el final de la cópula, ha sido también descrita para A. gazella, - Paulian, op. cit. -, A. forsteri, - Miller, 1974 -, Neophoca y Phocarctos -, Marlow op. cit. -

\section{REACCIONES DE BIENESTAR INTERPRETABLES COMO TERMORREGULATORIAS}

En Otaria flavescens se advierten los siguientes fenómenos fisiolóficos $\mathrm{y} / \mathrm{o}$ etológicos probablemente relacionados con termorregulación:

- jadeo

- lagrimeo y pérdida de saliva

- micción sobre el cuerpo, o mojado del cuerpo en lugares con orina

- exposición al aire de miembros extendidos

- extensión escrotal

- espolvoreo del cuerpo con arena de debajo de la superficie en que reposan 
- baños en el mar

- modificaciones de la distancia interindividual

- abanicado con el miembro posterior (excepcional).

En Arctocephalus australis hemos registrado:

- jadeo

- lagrimeo y pérdida de saliva

- uso de la sombra proyectada por rocas, realizado con gran efectividad tanto por cachorros como por juveniles y adultos

- concurrencia al agua, que se produce ante un aumento de la temperatura; en este caso se forman agrupaciones de lobos en el agua, que exponen los miembros al aire, en una gran variedad de posiciones

- En tierra fime extensión al aire de los miembros (poco frecuente), mecanismo regular para la evasión térmica en $A$. pusillus.

No hemos registrado nunca en $A$. australis el abanicado, actividad normal en Callorhinus ursinus; la emergencia escrotal; ni tampoco cambios de la distancia interindividual en grupos estables.

Aparte de los comportamientos de bienestar mencionados, probablemente relacionados con termorregulación, corresponde señalar otros comportamientos de bienestar presentes en las dos especies:

- rascado y acicalamiento de la piel de la cabeza y partes anteriores y dorso del cuello con las uñas de los miembros posteriores; de los flancos del cuerpo con el borde posterior de los miembros anteriores; de la base de los miembros posteriores con los dientes; y de diversas áreas del dorso, nuca, y eventualmente mentón, mediante movimientos laterales de frotamiento sobre el sustrato

- bostezo

- estornudo

- resoplo nasal

- eliminación del agua del pelaje, en tierra, por sacudidas al llegar del agua, o como ocurre en $A$. australis, al emerger del agua, mediante sacudidas de la cabeza y el cuello

- movimientos de compresión del pelaje, efectuados en el agua, particularmente de la cara, y en los que se utilizan simultáneamente los miembros anteriores.

\section{CONSIDERACIONES}

En poblaciones estudiadas de distintas especies de Otariidae, se advierte que los comportamientos tienen las siguientes particularidades: en algunos repertorios (por ej. rascado), las expresiones son muy similares en los distintos casos.

En lo que se refiere a territorialidad, reproducción y lucha, aunque muchos patrones son comunes, la diversificación tanto desde el punto. de vista cualitativo como cuantitativo, es marcada. 
En lo relativo a termorregulación es donde se evidencian las mayores diferencias, habiéndose desarrollado repertorios diferentes que probablemente conduzcan a fines semejantes.

Es muy posible que las diferencias registradas se deban en parte, $\mathrm{y}$ en algunos casos, a diferentes metodologías de estudio $\mathrm{y}$ de interpretación de los datos, pero es evidente que, fundamentalmente, reflejan ya particularidades específicas adquiridas en el curso de la evolución, ya respuestas a diferencias ecológicas en los habitats ocupados por las diferentes especies o poblaciones estudiadas.

Consideramos recomendable realizar estudios comparativos en diferentes poblaciones de una misma especie en situaciones ecológicas distintas, a fin de aclarar la naturaleza específica o poblacional de las diferencias etológicas presentes.

\section{REFERENCIAS}

Bartholomew, G., Jr. \& P. G. Hoel, 1953. Reproductive behavior of the Alaska fur seal (Callorhinus ursinus). J. Mammal. 34 (4): 417-436.

Gentry, R. L., 1975. Comparative social behavior of eared seals. Rapp. Proc.-Verb. Réun. Cons. int. Expl. Mer 169: 189-194.

Marlow, B. J., 1975. The comparative behavior of the Australasian sea lions Neophoca cinerea and Procarctos hookeri (Pinnipedia: Otariidae). Mammalia 39 (2): 159-230.

Miller, E. H., 1974. Social behavior between adult male and female New Zeland fur seals, Arctocephalus forsteri (Lesson), during the breeding season. Austr. J. Zool. 22: 155-173.

Miller, E. H., 1975. Social and evolutionary implications of territoriality in adult. male New Zeland fur seals, Arctocephalus forsteri (Lesson 1828), during the breeding season. Rapp. Proc.-verb. Réun. Cons. int. Expl. Mer 169: 170-187.

Paulian, P., 1964. Contribution à l'étude de l'otarie de l'île Amsterdam. Mammalia 28 (Supl. 1): 1-146.

Peterson, R. S. \& G. A. Bartholomew, 1967. The natural history and behavior of the California sea lion. Am. Soc. Mammal. Spec. Publ. 1: 1-79.

Vaz-Ferreira, R., 1981. South American sea lion, Otaria flavescens (Shaw, 1800), pp. 39-65, in S. H. Ridgway \& R. J. Harrison, eds., Handbook of marine mammals 1. Academic Press, London \& New York.

Vaz-Ferreira, R. \& S. Vallejo, 1981. Algunos aspectos del comportamiento de Arctocephatus australis (Zimmerman), lobo de los pelos de Sudamérica (Pinnipedia: Otariidae) en el Uruguay. Symp. VI Jorn. argent. Zool.: 223-236. 

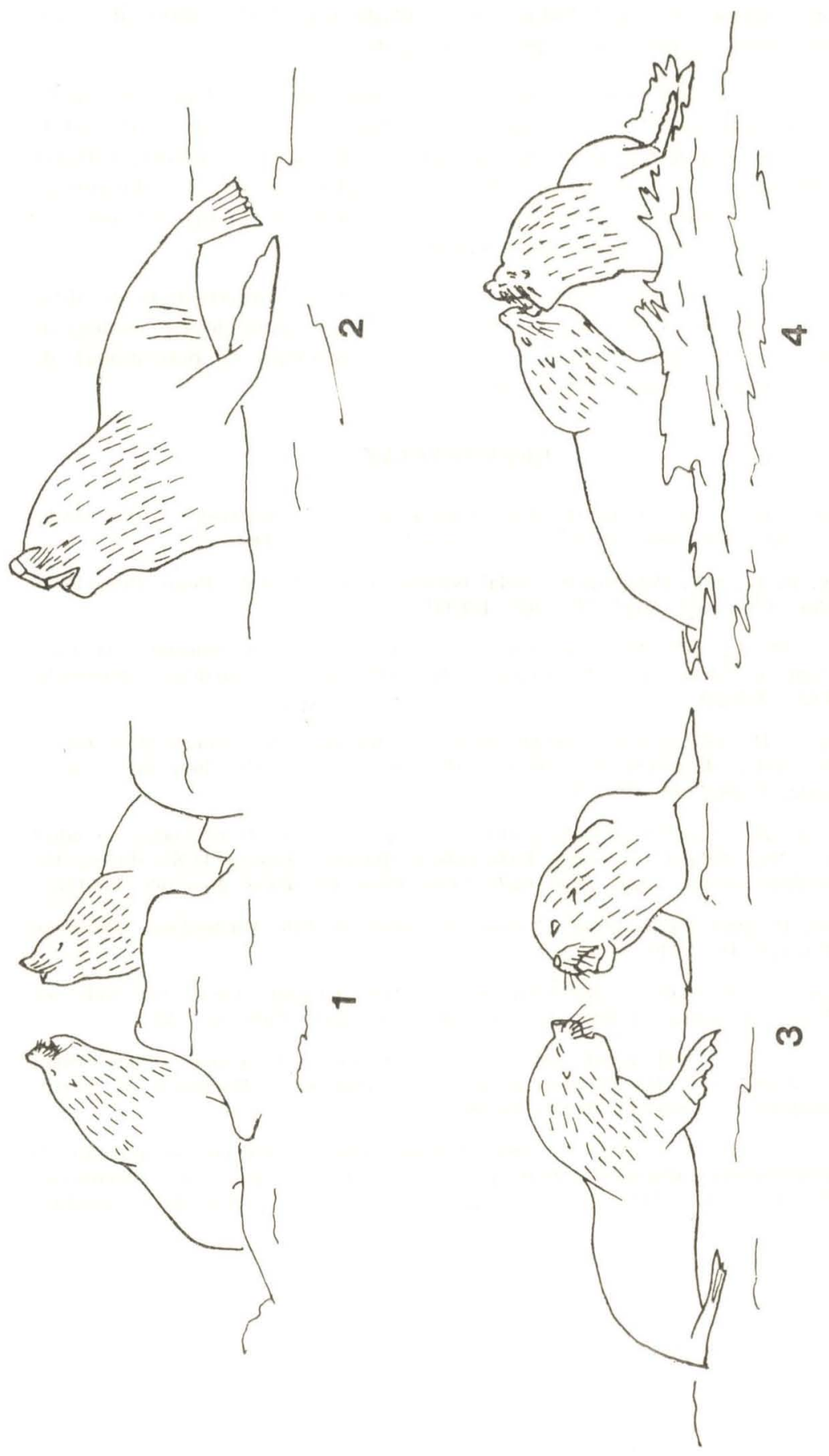

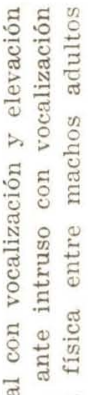

胥。

Ф.

댕

है के

造

ङ छ

-1 0

ฟิ

สี ฐี ซี

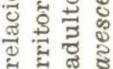

का

త

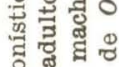

เั

के द्ञ

$\approx$ घ

|

을

खैं के 조

>芯萦

m

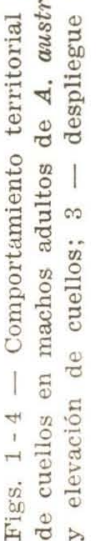



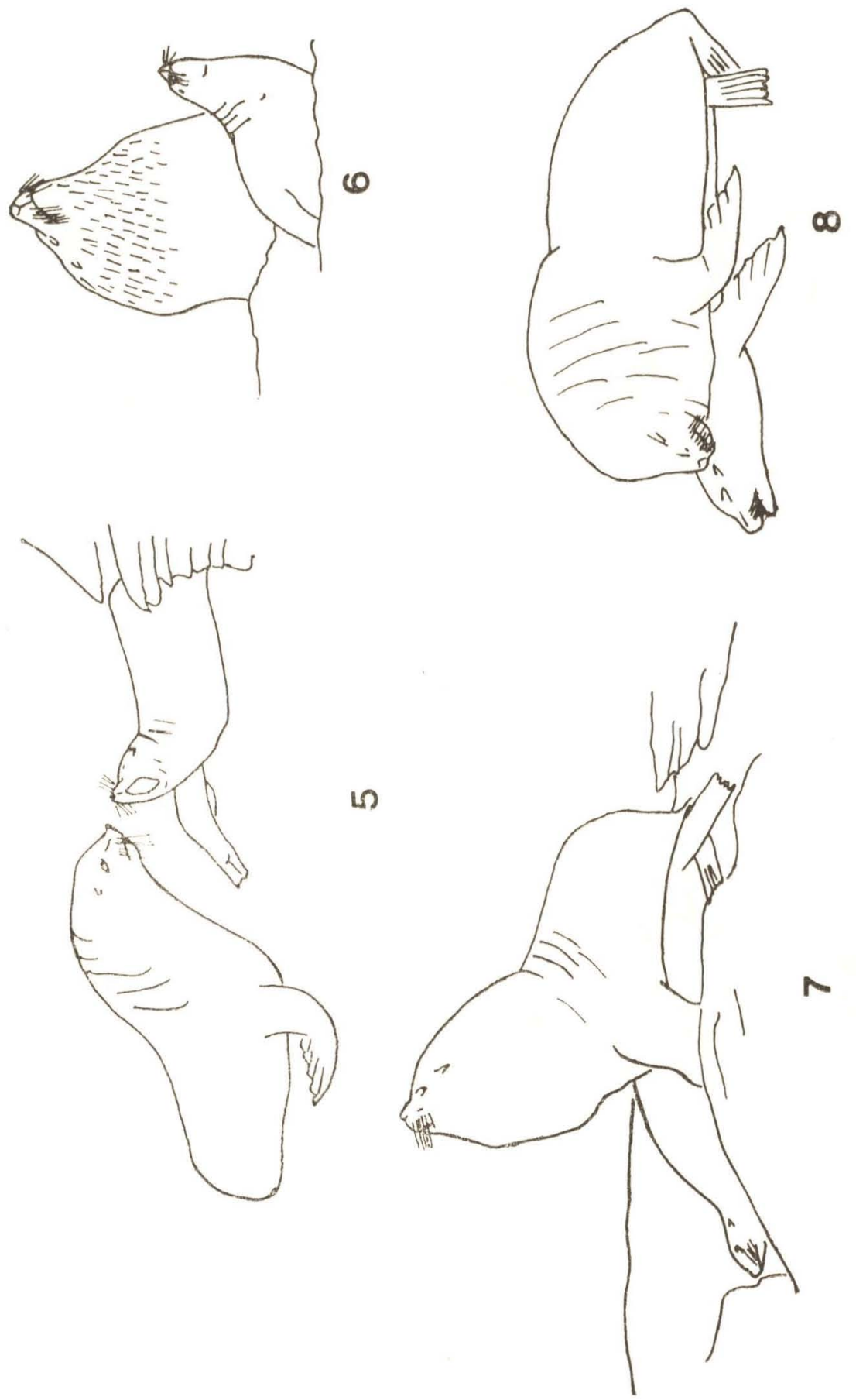

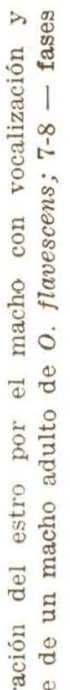

苋瀶 성 ช) इ 跑 造 풍 \pm क्ष | 몽 10.

菅 


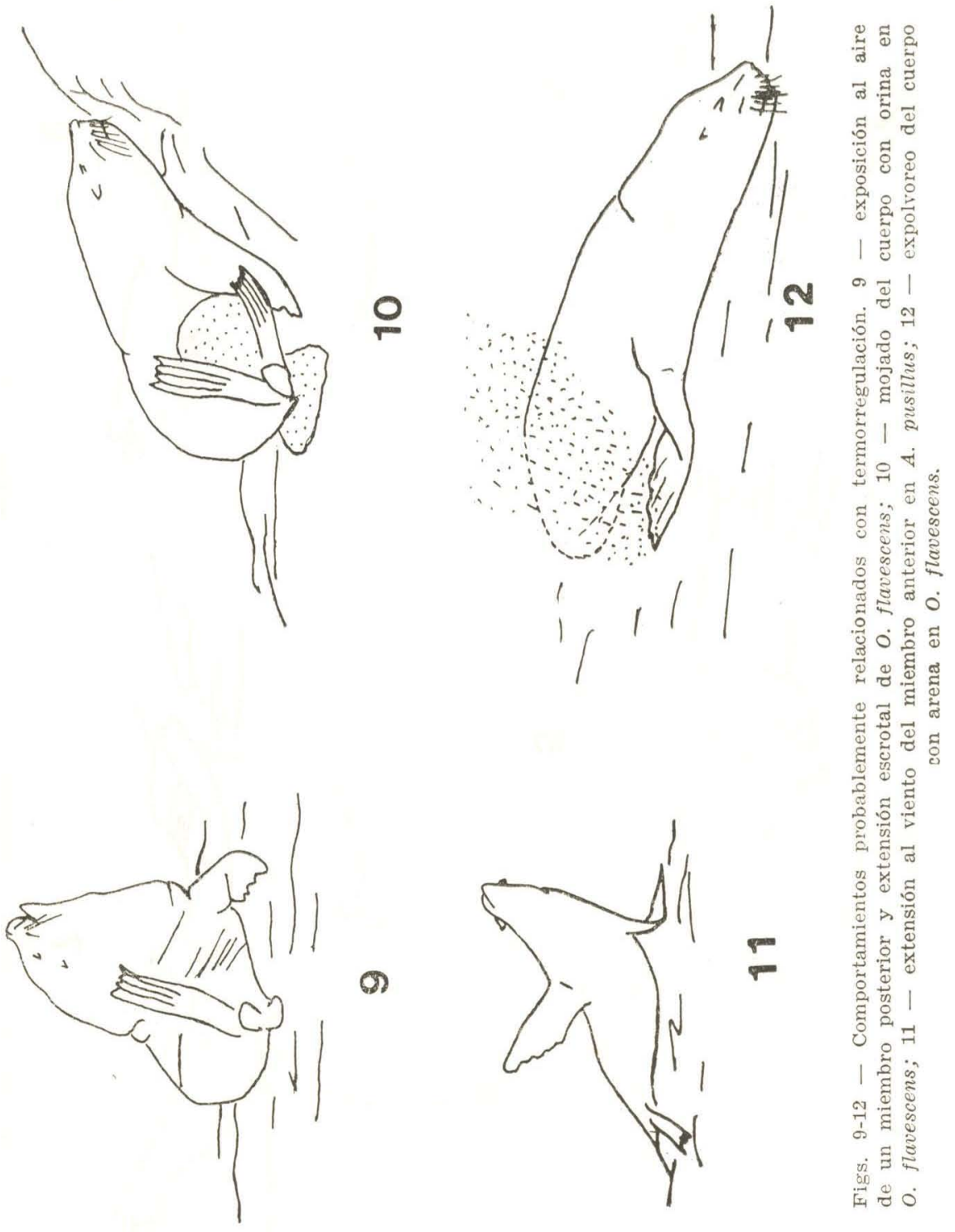

